\title{
Ordering Physician
}

National Cancer Institute

\section{Source}

National Cancer Institute. Ordering Physician. NCI Thesaurus. Code C54089.

The physician who has ordered diagnostic tests, services, items (e.g., durable medical equipment), or supplies for a patient or study subject. 